\title{
PH Model-based Shape Reconstruction of Heterogeneous Continuum Closed Loop Kinematic Chain: An Application to skipping Rope
}

\author{
Inderjeet Singh ${ }^{1}$, Yacine Amara ${ }^{2}$, Othman Lakhal ${ }^{1}$, Achille Melingui ${ }^{3}$ and Rochdi Merzouki ${ }^{1}$
}

\begin{abstract}
Soft robotics is a swiftly growing research area these days. Modeling continuum robots accurately is still a demanding field. The paper aims to propose a shape reconstruction method and the estimation of the kinematic behavior of heterogeneous continuum robot in closed loop kinematic configuration, by using Pythagorean Hodograph (PH) curves. The validation of the model approach has been tested on cooperative continuum robots, namely Compact Bionic Handling Arms (CBHA), driving an intermediate flexible rope (a passive flexible link), by using a 3D tracking system. Experimental comparison of the proposed approach with the existing approaches is performed in terms of accuracy as well as the time cost.
\end{abstract}

\section{INTRODUCTION}

Continuum robots are made up of soft material to produce a flexible motion. These robots can be inspired from nature (biological activities) like an elephant's trunk [1], a fish or an octopus [2]. They are complex to model due to their nonlinear behavior and high degree of freedoms.

Various models for continuum manipulators are studied in terms of kinematics and shape reconstruction. A noval approach based on shape functions is proposed to model spatial kinematics of a multiple section continuum manipulator [3], assuming constant curvature. In this approach appropriate shape functions are chosen to remove the singularity [4]. Kinematic modeling of Bionic Handling Assistant (BHA) is done by representing the manipulator as torus segments [5]. Various Forward kinematic models of CBHA manipulators are discussed and compared in terms of accuracy in [6]. In [7], OctArm V manipulator consisting of air muscle actuators is modeled geometrically using cosserat rod theory and a fibre reinforced model. Inverse kinematic model (IKM) of a continuum manipulator is described using feed-forward neural networks in [8]. In [9], Neural Networks (NN) approach is used to approximate the IKM of the CBHA manipulator. A hybrid approach (quantitative as well as qualitative) [10] is used for IKM of CBHA manipulator in which a geometrical approach is used to compute the inverse kinematic equations of the CBHA manipulator and then $\mathrm{NN}$ is used to solve these equations due to their high non-linearity. In [11], various IKM models are compared for CBHA manipulator in terms

1 I. Singh, O. Lakhal and R. Merzouki are with Polytech Lille, CRIStAL, CNRS-UMR 9189, Avenue Paul Langevin, 59655 Villeneuve d'Ascq, France inderjeet.singh@ed.univ-lillel.fr rochdi.merzoukiepolytech-lille.fr othmen11@hotmail.fr

${ }^{2}$ Y. Amara is with Ecole Militaire Polytechnique, BP 17, Bordj EL Bahri, Algiers 16111, Algeria amara.yacine@gmail.com

3 A. Melingui is with the Ecole Nationale Superieure Polytechnique, University of Yaounde 1, Yaounde 8390, Cameroon achillemelingui@gmail.com of accuracy and time cost.

Furthermore, there are many works in which sensors are used to reconstruct the shape of the continuum manipulators [12], [13], [14]. In [15], a correspondence between a hyperredundant manipulator and a geometric curve has been established by solving a non-linear optimization problem called 'shape inverse problem'. The backbone shape of a hyper-redundant manipulator has also been solved using the modal approach and thereby using a fitting algorithm to join the various sections of a discrete 30 DOF manipulator [16]. Pythagorean Hodograph curves and their applications are defined by Farouki and Sakkalis in 1990 [17]. These curves have the following main properties: 1) Length as well as bending energy of these curves can be calculated in the closed form 2) Easy to construct parallel curves 3) Only four boundary conditions are required to construct a quintic $\mathrm{PH}$ curve: starting position, final position and direction vectors at both of these positions. In robotics field, PH curves are used mainly for the trajectory planning purposes. $\mathrm{PH}$ curves have been used in 3D path planning of three unmanned aerial vehicles (UAVs), as well as to avoid obstacles in their path [11]. In [18], PH curves are utilized to model spatial continuum manipulators for the first time, which is being extended in this paper. In this paper, work is focused on the concatenation of more than one $\mathrm{PH}$ curves to model more complex shapes of continuum manipulator or to model continuum closed loop kinematic chains. The main contributions of this work are:

- Concatenation of Pythagorean Hodograph curves to model either serial continuum manipulators or the continuum closed loop kinematic chains.

- Building a heterogeneous continuum closed loop kinematic chain using two CBHA robots and a rope (passive flexible link). Heterogeneous means the use of different kind of links in a chain: as CBHA and the rope in the presented case.

- Experimental validation of the PH based model for the continuum closed loop kinematic chain.

- PH curves can be used to model heterogeneous links in a kinematic chain.

The organization of the paper is as follows: Section II describes the Pythagorean hodograph condition and also the generation of the $\mathrm{PH}$ curves. Concatenation of $\mathrm{PH}$ curves is discussed in section III to model continuum closed loop kinematic chains. Section IV describes the experimental set -up and the experimental validations of the proposed methodology. Results and discussions are presented in section V. 
Finally, the conclusions, as well as future perspectives of the presented work, are discussed in section VI.

\section{Pythagorean Hodograph In Context of CONTINUUM MANIPULATORS}

\section{A. Shape Reconstruction of Continuum Manipulators or a flexible link}

Continuum manipulators are continuously curving manipulators to produce a flexible motion. Pythagorean Hodograph curves are used in the context of continuum manipulators to reconstruct their optimal posture [18]. Let us consider two spatial poses $P_{s}$ and $P_{f}$ on the continuum manipulator as:

$$
\begin{array}{r}
P_{s}\left(x_{s}, y_{s}, z_{s}, \alpha_{s}, \beta_{s}, \gamma_{s}\right) \\
P_{f}\left(x_{f}, y_{f}, z_{f}, \alpha_{f}, \beta_{f}, \gamma_{f}\right)
\end{array}
$$

Here, $P_{s}$ is the starting pose of the continuum manipulator located at its base. $P_{f}$ is the final pose of the continuum manipulator located at its end. $\mathrm{PH}$ curve is used to reconstruct the shape of the continuum manipulator from its starting pose to the final pose. PH curves are the normal polynomial curves with an extra condition of Pythagorean Hodograph. Therefore, these curves are the special case of the normal polynomial curves and are explained as follows [19]:

Let $r(h)$ be the normal polynomial curve used to reconstruct the shape of the continuum manipulator.

$$
r(h)=(x(h), y(h), z(h)) ; \quad 0 \leq h \leq 1
$$

$h$ be the normalized curvilinear coordinate of the curve. It means:

$$
\begin{aligned}
P_{s} & =r(0)=(x(0), y(0), z(0)) \\
P_{f} & =r(1)=(x(1), y(1), z(1))
\end{aligned}
$$

Hodograph is a term defined as the first derivative of the curve $r(h)$. It is parallel to the tangent to the curve. It is given as:

$$
r^{\prime}(h)=\left(x^{\prime}(h), y^{\prime}(h), z^{\prime}(h)\right)
$$

The length $L(h)$ of the continuum manipulator reconstructed using curve $r(h)$ is:

$$
L(h)=\int_{0}^{1}\left|r^{\prime}(h)\right| \mathrm{d} h=\int_{0}^{1} \sqrt{x^{\prime}(h)^{2}+y^{\prime}(h)^{2}+z^{\prime}(h)^{2}} \mathrm{~d} h
$$

Equation (5) represents the length of the continuum manipulator. Due to the presence of the square root in the integral, the closed form solution of the length is not possible. Therefore, a numerical method is needed to find the approximation of the actual solution. To get rid of this situation, the square root sign can be replaced, if:

$$
x^{\prime}(h)^{2}+y^{\prime}(h)^{2}+z^{\prime}(h)^{2}=\sigma(h)^{2}
$$

This is called the Pythagorean law or condition in three dimensions. Therefore,

The first derivatives (Hodographs) of parametric polynomials which satisfy the Pythagorean condition, are known as Pythagorean Hodographs.

Now length can be calculated in closed form as:

$$
L(h)=\int_{0}^{1}|\sigma(h)| \mathrm{d} h
$$

A sufficient and necessary condition which satisfies eq. (6) is given in [20], [19], i.e. the four polynomials $u(h), v(h), p(h)$ and $q(h)$ are used to express the hodograph components as:

$$
\begin{gathered}
x^{\prime}(h)=\left[u^{2}(h)+v^{2}(h)-p^{2}(h)-q^{2}(h)\right] \\
y^{\prime}(h)=2[u(h) q(h)+v(h) p(h)] \\
z^{\prime}(h)=2[v(h) q(h)-u(h) p(h)]
\end{gathered}
$$

$u(h), v(h), p(h)$ and $q(h)$ polynomials are chosen so that $\sigma(h)$ is:

$$
\sigma(h)=u^{2}(h)+v^{2}(h)+p^{2}(h)+q^{2}(h)
$$

Generalized degree of PH curves is $2 n+1$, where $n$ is the degree of polynomials $u(h), v(h), p(h)$ and $q(h)$. The lowest degree PH curves are cubic PH curves while linear polynomials are used. Cubic PH curve does not have any free control point (out of four control points), it means they have a unique solution only. Therefore, cubic PH curves are not good for shape representation. Quintic PH curves can be generated by using quadratic polynomials $u(h), v(h), p(h)$ and $q(h)$. Quintic PH curves contain two free control points which makes them more suitable to reconstruct the actual shapes accurately [19]. Hence, the following development uses the quintic PH curves to model continuum manipulators. The expression of quintic $\mathrm{PH}$ curve is derived by using quadratic polynomials $u(h), v(h), p(h)$ and $q(h)$ in the Bernstein form [21], [19]. These quadratic polynomials are inserted in eqs. $(8,9,10)$ and integrated from both sides to yield the general form of quintic PH curve $r(h)$ :

$$
r(h)=\left[\begin{array}{l}
x(h) \\
y(h) \\
z(h)
\end{array}\right]=\sum_{k=0}^{5} P_{k}\left(\begin{array}{l}
5 \\
k
\end{array}\right)(1-h)^{5-k} h^{k}
$$

It implies,

$$
r(h)=\left[\begin{array}{l}
1 \\
h \\
h^{2} \\
h^{3} \\
h^{4} \\
h^{5}
\end{array}\right]^{T}\left[\begin{array}{cccccc}
1 & 0 & 0 & 0 & 0 & 0 \\
-5 & 5 & 0 & 0 & 0 & 0 \\
10 & -20 & 10 & 0 & 0 & 0 \\
-10 & 30 & -30 & 10 & 0 & 0 \\
5 & -20 & 30 & -20 & 5 & 0 \\
-1 & 5 & -10 & 10 & -5 & 1
\end{array}\right]\left[\begin{array}{c}
P_{0} \\
P_{1} \\
P_{2} \\
P_{3} \\
P_{4} \\
P_{5}
\end{array}\right]
$$

The derived form is the Bernstein-Bezier form. Here, $P_{k}=$ $\left(x_{k}, y_{k}, z_{k}\right)$ are the control points. These control points are in terms of $u(h), v(h), p(h)$ and $q(h)$. Therefore next step is to find these optimal polynomials $u(h), v(h), p(h)$ and $q(h)$ to calculate the control points $P_{k}$.

Basic Schematic of a quintic PH curve to reconstruct the shape of the continuum manipulator is shown in Fig. $1 . \vec{d}_{s}$ and $\vec{d}_{f}$ are the direction vectors at starting (base) and ending point of continuum manipulator respectively. They can be computed using orientations $\left(\alpha_{s}, \beta_{s}, \gamma_{s}\right)$ and $\left(\alpha_{f}, \beta_{f}, \gamma_{f}\right)$. Using four conditions $P_{s}, d_{s}, P_{f}$ and $d_{f}$, polynomials $u(h), v(h)$, $p(h)$ and $q(h)$ are calculated which leads to the calculation of the control points as in [18]. Due to the presence of two free control points, there are infinite solutions in case of quintic PH curves. Therefore an optimal solution is selected with 


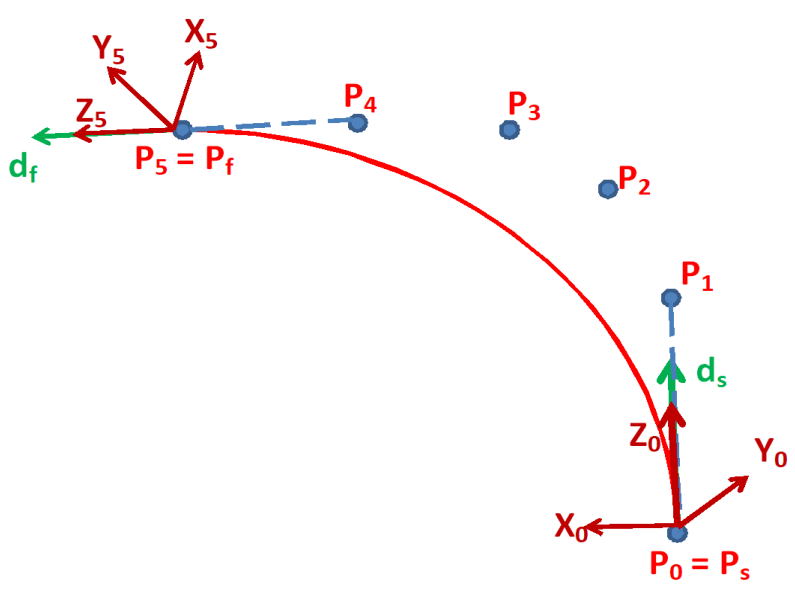

Fig. 1. Basic schematic of PH curve

minimum potential energy as discussed in [18] to reconstruct the shape of the continuum manipulator. Because a physical system always tries to move from one point to the other point with minimum potential energy (combination of bending and twisting energy).

\section{B. Inverse Kinematics of Continuum Manipulators}

Shape reconstruction of continuum manipulators using $\mathrm{PH}$ curves leads to the inverse kinematics of the manipulator. In case of continuum manipulators, IKM is the relationship between the coordinates of the tip of the manipulator, i.e., the end point of $\mathrm{PH}$ curve and its length respectively. By combining Eq. (13) and Eq. (5), the following expression of $\mathrm{L}(\mathrm{h})$ is obtained.

$$
\begin{aligned}
L(h)= & \int_{0}^{1}\left(\left(-5+20 h-30 h^{2}+20 h^{3}-5 h^{4}\right) P_{0}\right. \\
& +\left(5-40 h+90 h^{2}-80 h^{3}+25 h^{4}\right) P_{1} \\
& +\left(20 h-90 h^{2}+120 h^{3}-50 h^{4}\right) P_{2} \\
& +\left(30 h^{2}-80 h^{3}+50 h^{4}\right) P_{3}+\left(20 h^{3}-25 h^{4}\right) P_{4} \\
& \left.+\left(5 h^{4}\right) P_{5}\right) d h
\end{aligned}
$$

Therefore, solving eq. (14) in the matrix form, the length of the backbone of the continuum manipulator can be computed [18].

\section{Concatenation of PH CURVes to BUild A CONTINUUM CLOSED LOOP KINEMATIC CHAIN}

Concatenation of more than one $\mathrm{PH}$ curves is necessary in the following two cases:

\section{A. Case 1:}

Sometimes the continuum manipulators are difficult to model with one $\mathrm{PH}$ curve due to their complex shape or due to the more number of sections. Also in the case of continuum closed loop kinematic chains, to model more than one link, concatenation of PH curves is required. As shown in Fig. 2, two PH curves are concatenated. In this case, both of the links are joined with $C_{1}$ continuity. Therefore this is the case in which position, as well as velocity, are same at the junction point. Following conditions need to be used to construct $\mathrm{PH}$ curves for this case:

For $\mathrm{PH}_{1}$,

$$
\begin{array}{cc}
r_{1}(0)=A & r_{1}(1)=B \\
r_{1}^{\prime}(0)=d_{s} & r_{1}^{\prime}(1)=d_{m}
\end{array}
$$

For $\mathrm{PH}_{2}$,

$$
\begin{array}{cc}
r_{2}(0)=B & r_{2}(1)=C \\
r_{2}^{\prime}(0)=d_{m} & r_{2}^{\prime}(1)=d_{f}
\end{array}
$$

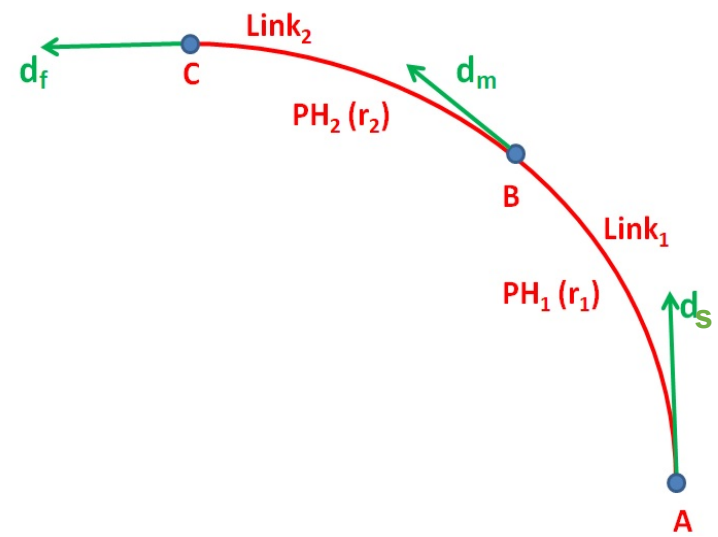

Fig. 2. Concatenation of two PH curves with $C_{1}$ continuity

\section{B. Case 2:}

In a continuum kinematic chain, sometimes the links are joined with $C_{0}$ continuity having only the position constraint at the junction. Fig. 3 shows the concatenation of two $\mathrm{PH}$ curves (two links) with $C_{0}$ continuity. Following conditions need to be used to construct $\mathrm{PH}$ curves for this case:

For $\mathrm{PH}_{1}$

$$
\begin{array}{cc}
r_{1}(0)=A & r_{1}(1)=B \\
r_{1}^{\prime}(0)=d_{s} & r_{1}^{\prime}(1)=d_{f}
\end{array}
$$

For $\mathrm{PH}_{2}$,

$$
\begin{array}{cc}
r_{2}(0)=B & r_{2}(1)=C \\
r_{2}^{\prime}(0)=d_{s s} & r_{2}^{\prime}(1)=d_{f f}
\end{array}
$$

\section{EXPERIMENTAL TESTS}

An OptiTrack vision system (Figs. 4,5) is used for all of the experimental validations. Four Prime13 cameras are used for tracking, each of them having configuration:

- Resolution: 1.3 MP $(1280 \times 1024)$

- Frame rate: 240 FPS

- Filter Switcher: Included

- Interface: GigE/PoE

- No. of LEDs: 62

- Latency: $4.2 \mathrm{~ms}$ 


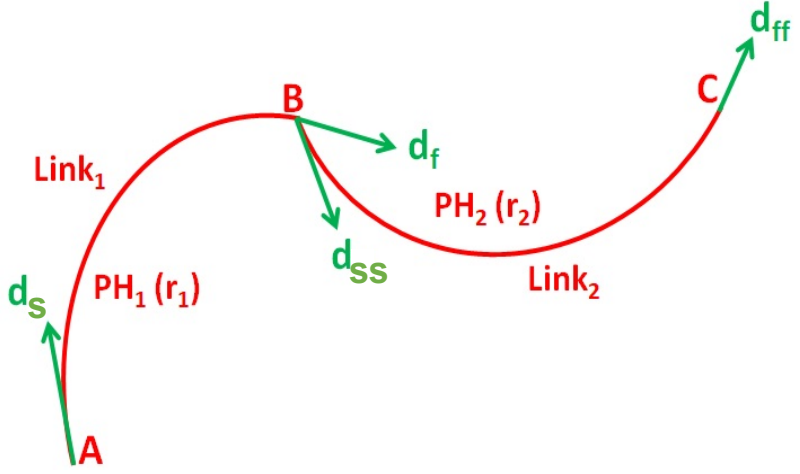

Fig. 3. Concatenation of two PH curves with $C_{0}$ continuity

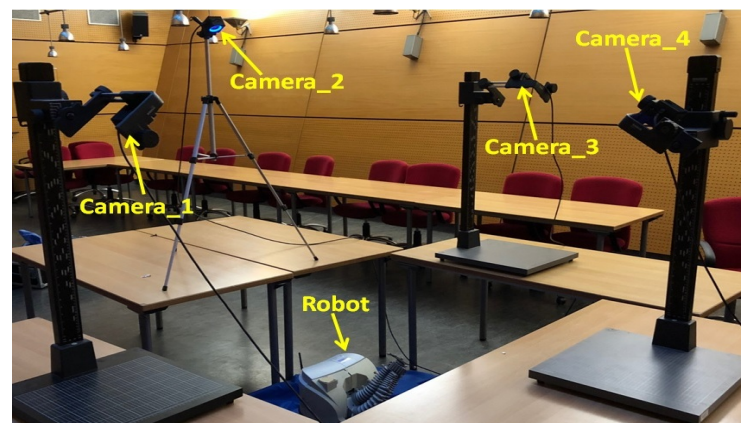

Fig. 4. Experimental Set-up

Motive 2.0 software is used for this OptiTrack vision system.

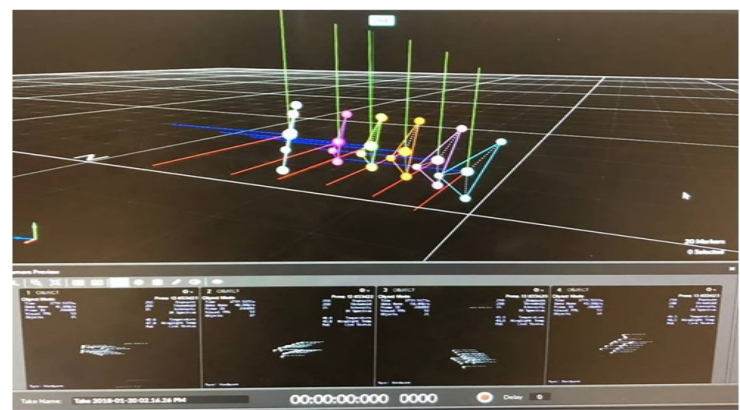

Fig. 5. Motive 2.0 software for tracking of shape of CBHA arm

\section{A. Modeling One CBHA Arm using PH-curve (Open Loop Configuration Case)}

The proposed $\mathrm{PH}$ curves modeling method is applied to Compact Bionic Handling Assistant (CBHA) manipulator of Fig. 6, designed by Festo [22]. CBHA manipulator, also called elephant trunk manipulator, is made up of a soft elastic polyamide material, where it is divided into two sections, a rotating wrist and a compliant gripper (Fig. 6). Each section is composed of three tubes, connected each other through a backbone, and actuated by electro-pneumatic actuators.
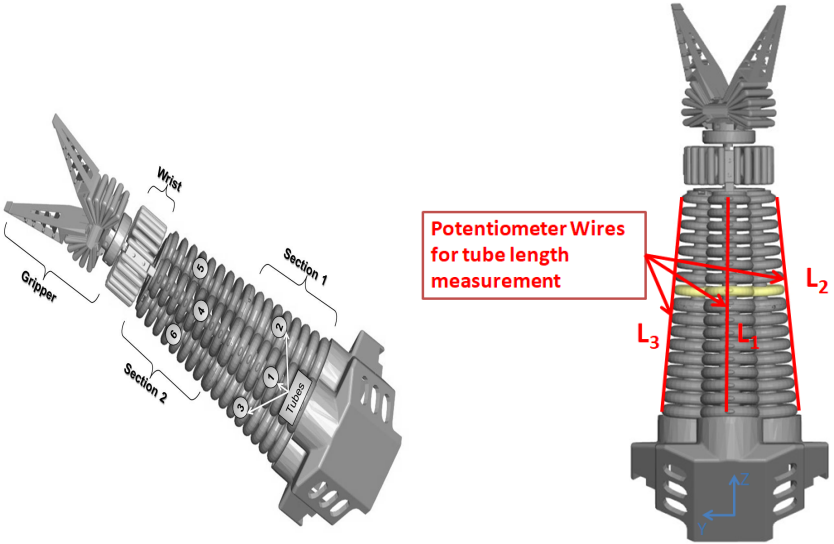

Fig. 6. CBHA manipulator description

Pressure change inside the tubes leads to change in their respective lengths, inducing the change in the position and orientation of the tip of the manipulator.

Equal pressures in all three tubes maintain the robot in straight line longitudinal posture. An inextensible cable placed at the backbone of the manipulator limits the maximum extension. To create bending, differential pressures can be applied inside the tubes. The elongation of each tube is measured with a wire-potentiometer. There are six wirepotentiometers, three of them measure the tube lengths of the first section, and the three others measure the total tube lengths. CBHA is not able to make a torsion movement around its longitudinal axis. Thus, no twisting behavior can be generated during its displacement.

In the following development, it is supposed that the two bending sections (Fig. 6) are fused to one entire continuum section, without any shape disconnection. As discussed in section II, the proposed modeling method shows how the backbone shape of the CBHA can be reconstructed by using $\mathrm{PH}$-curves and then how its IKM can be deduced.

To perform the experimental validation, five markers are

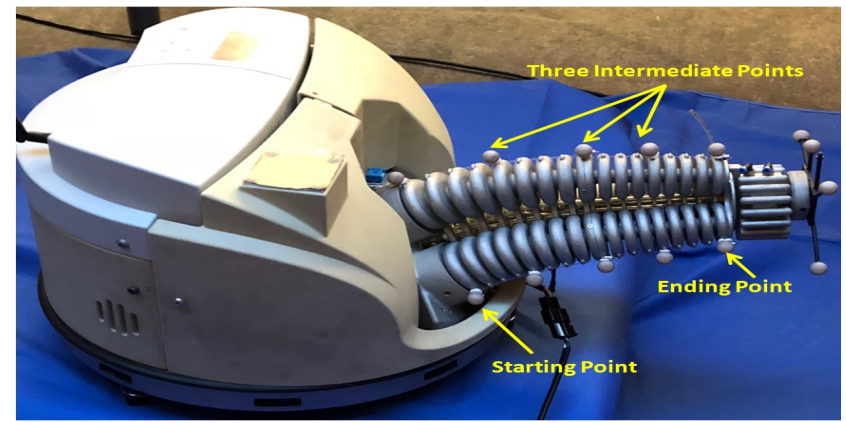

Fig. 7. Shape tracking of CBHA arm

attached to each tube of the CBHA arm (Fig. 7): at starting point, at ending point and three intermediate points. Inputs are given to CBHA arm to create a random posture. Using vision system, the data of the markers is tracked. Four inputs to construct a $\mathrm{PH}$ curve for CBHA arm are: starting 
point, direction vector at the starting point, ending point and direction vector at the ending point. Therefore, a $\mathrm{PH}$ curve is constructed from starting point to the ending point of the CBHA arm.

\section{B. Closed Loop Kinematic Chain of Continuum Links}

A heterogeneous continuum closed loop kinematic chain is formed to move towards the application of skipping rope. A CBHA manipulator can move an object like we can move it by hand. Therefore, A heterogeneous continuum closed loop kinematic chain is formed with an assembly of three flexible links. Fig. 8 shows the two CBHA arms driving an intermediate flexible rope, forming a closed kinematic chain $\mathrm{ABCD}$. The rope is a passive link, and it is hanging under the condition of its self-weight. Both of the CBHA arms are fixed at their bases. The aim is to model the shape of heterogeneous continuum kinematic chain using Pythagorean Hodograph curves.

Let us consider that $C B H A_{1}$ acts as a $l i n k_{1}$, rope as a $l i n k_{2}$ and $C B \mathrm{HA}_{2}$ as a link $_{3}$. A PH curve is used to reconstruct the shape of each flexible link of the closed kinematic chain (Fig. 9). Table I shows the four initial conditions used to construct the PH curve for each link. Therefore, three PH curves are constructed for the considered kinematic chain.

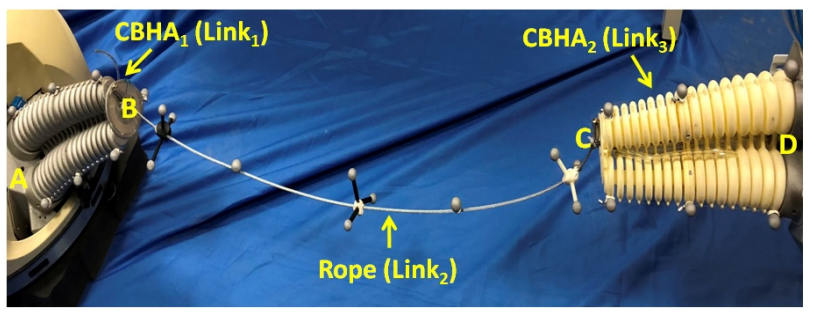

Fig. 8. Heterogeneous continuum closed loop kinematic chain

TABLE I

INITIAL CONDITIONS TO CONSTRUCT PH CURVES FOR HETEROGENEOUS CONTINUUM CLOSED LOOP KINEMATIC CHAIN

\begin{tabular}{|c|c|c|c|}
\hline & Link $_{1}\left(\mathrm{PH}_{1}\right)$ & Link $_{2}\left(\mathrm{PH}_{2}\right)$ & Link $_{3}\left(\mathrm{PH}_{3}\right)$ \\
\hline Starting Position & $\mathrm{A}$ & $\mathrm{B}$ & $\mathrm{C}$ \\
\hline Starting Orientation & $O(A)_{C B H A_{1}}$ & $O(B)_{\text {Rope }}$ & $O(\mathrm{C})_{\mathrm{CBHA}_{2}}$ \\
\hline Ending Position & $\mathrm{B}$ & $\mathrm{C}$ & $\mathrm{D}$ \\
\hline Ending Orientation & $O(\mathrm{~B})_{C B H A_{1}}$ & $O(\mathrm{C})_{\text {Rope }}$ & $O(\mathrm{D})_{\mathrm{CBHA}_{2}}$ \\
\hline
\end{tabular}

To do experimental validation, markers are attached on all of the three links of the closed kinematic chain: $C B H A_{1}$, rope and $\mathrm{CBHA}_{2}$ as shown in Fig. 8.

\section{RESUlts AND Discussions}

Results are presented for the following sections:

\section{A. Shape reconstruction of single CBHA arm using PH curve}

Fig. 10 shows a posture of CBHA arm as well as the reconstruction of the shape of the $\mathrm{CBHA}$ arm using $\mathrm{PH}$ curve. $\mathrm{PH}$ construction uses only start and the end point of the CBHA arm. Other three intermediate points are used for the comparison of the reconstructed shape with the actual

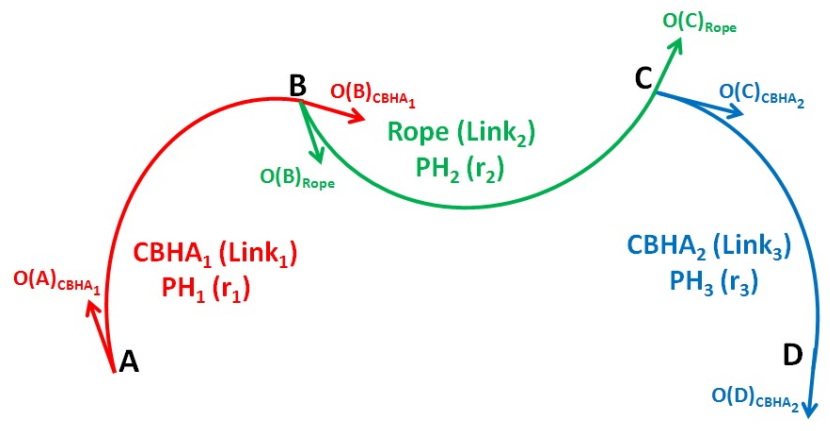

Fig. 9. Modeling of closed loop kinematic chain using PH curves

shape. In this case, the three intermediate points (actual shape) are at distances $1.6135 \mathrm{~mm}, 4.2048 \mathrm{~mm}$ and 3.8339 $\mathrm{mm}$ respectively from the reconstructed $\mathrm{PH}$ shape.

Furthermore, a trajectory (Fig. 11) with 100 points is

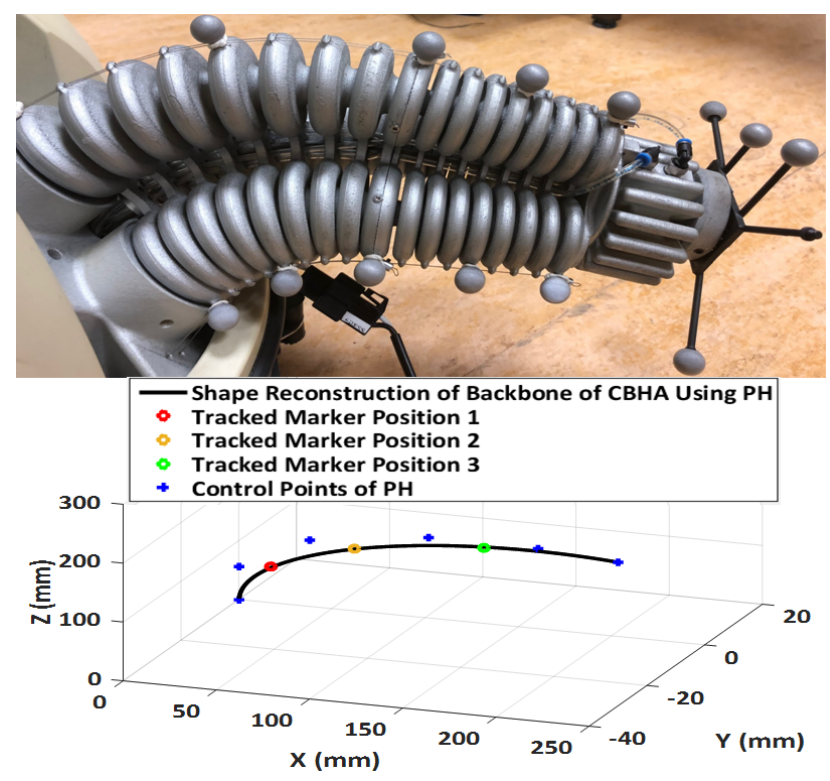

Fig. 10. Comparison of real shape with shape reconstructed using $\mathrm{PH}$ curve for single CBHA arm

recorded using vision system. Base of the CBHA manipulator is fixed. Therefore, Starting point as well as orientation at that point is fixed. Ending point as well as orientation at that point is used from the tracked trajectory (Fig. 11). PH curves methodology is used to reconstruct the shape of the backbone of the CBHA arm for the whole trajectory. Some random postures reconstructed using $\mathrm{PH}$ curves are shown in Fig. 12. During this trajectory, to compare the actual shape with the PH shape, the distances of the three intermediate tracked points is calculated from the $\mathrm{PH}$ shape. The average distances for the whole trajectory (100 postures) are $1.2 \mathrm{~mm}$, $3.7 \mathrm{~mm}$ and $3.1 \mathrm{~mm}$ respectively.

The errors in the shape reconstructed by $\mathrm{PH}$ curve are acceptable. It means PH curves can approximate the shape of the continuum manipulators. The reason of these small errors 


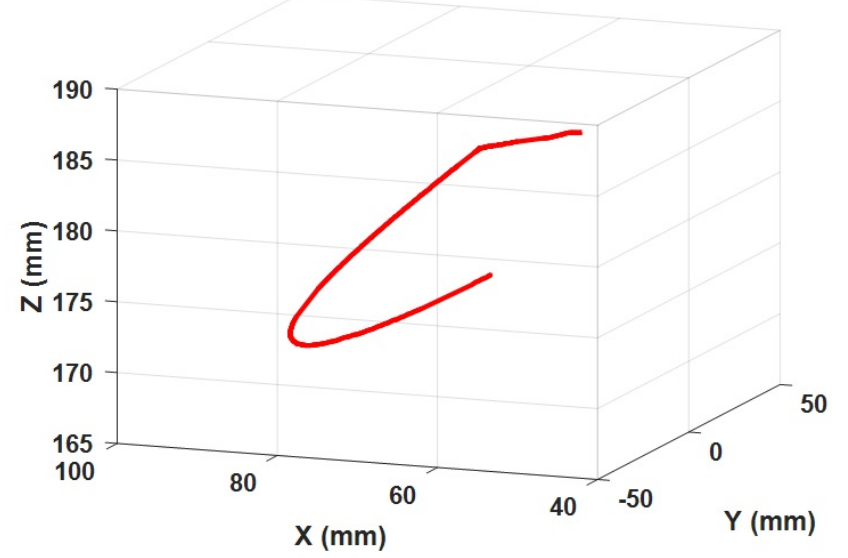

Fig. 11. Trajectory tracked using vision system

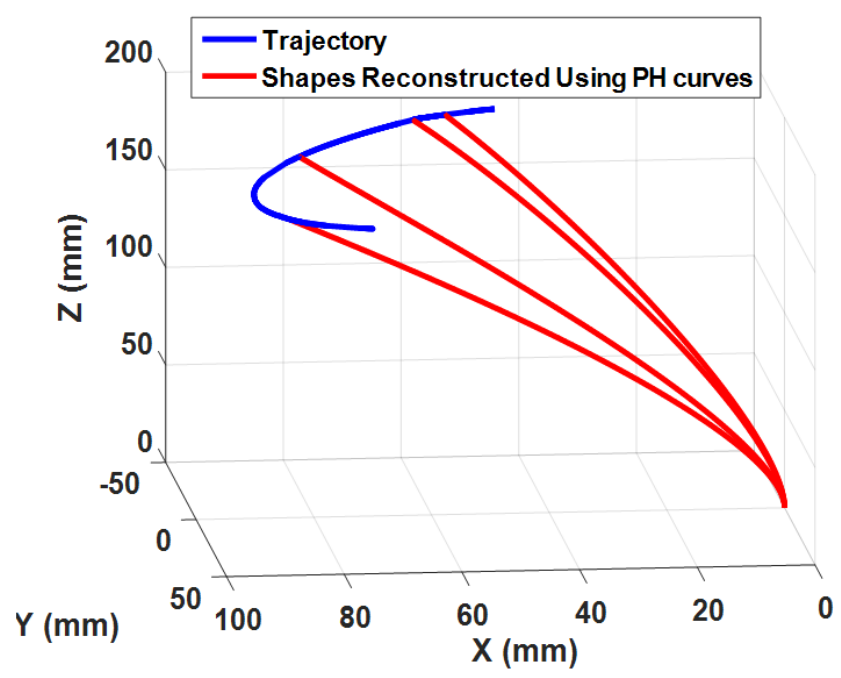

Fig. 12. Random postures reconstructed using $\mathrm{PH}$ curves along the trajectory

can be the choice of an optimal quintic PH curve from the multiple solutions. In future the focus would be to reduce these errors by modifying the choice of optimal quintic $\mathrm{PH}$ curve.

\section{B. Inverse kinematics of CBHA arm}

Length of the backbone of the CBHA arm is computed using PH method for the trajectory (Fig. 11). Three tubes are attached (constrained) to the backbone of the CBHA arm. Therefore the lengths $L_{1}, L_{2}$ and $L_{3}$ of the entire tubes of the CBHA arm are computed from the backbone length [18]. Lengths of the three tubes are also recorded from the potentiometer wire sensors of the arm as the reference lengths. Two inverse kinematic methods already exist for CBHA arm: 1) Hybrid Method [10] 2) Newton Raphson Method [11]. Therefore the proposed method, as well as existing methods, are compared with the reference lengths. The errors are being tabulated in table II. From the errors, it is confirmed that there is improvement in the accuracy of inverse kinematic solution using $\mathrm{PH}$ curves based method. Also, PH curves approach can approximate the shape of the CBHA manipulator, but other approaches can not. Table III shows the time cost of each of the method while the calculation of the inverse kinematics of the CBHA. PH curves approach is the most time efficient among other approaches.

\section{Closed Loop Kinematic Chain of Continuum Links}

Three PH curves are constructed for the kinematic chain as described in Fig. 9. The reconstructed shape of the closed kinematic chain using PH curves is compared with the actual tracked shape (Fig. 14). The average errors from the actual shape (tracked markers) for the links $C B H A_{1}$, rope and $\mathrm{CBHA}_{2}$ are $2.8 \mathrm{~mm}, 8 \mathrm{~mm}$ and $3.1 \mathrm{~mm}$ respectively. These results show that $\mathrm{PH}$ curves based approach can approximate the shape of the heterogeneous links in a chain. Different

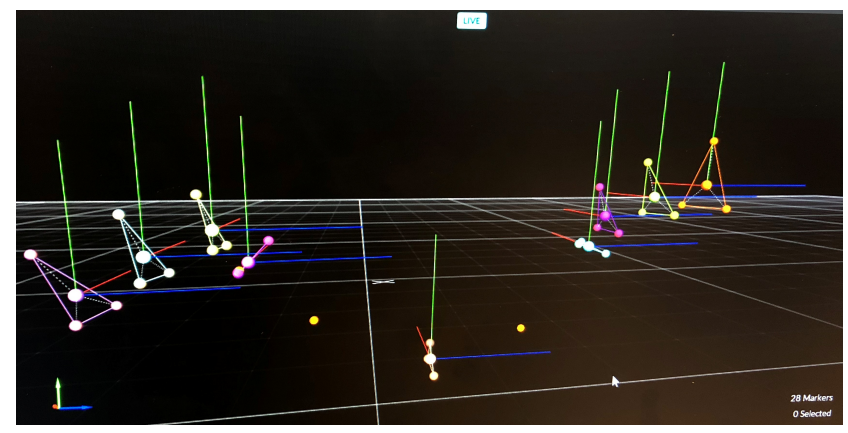

Fig. 13. Tracking of continuum closed loop kinematic chain
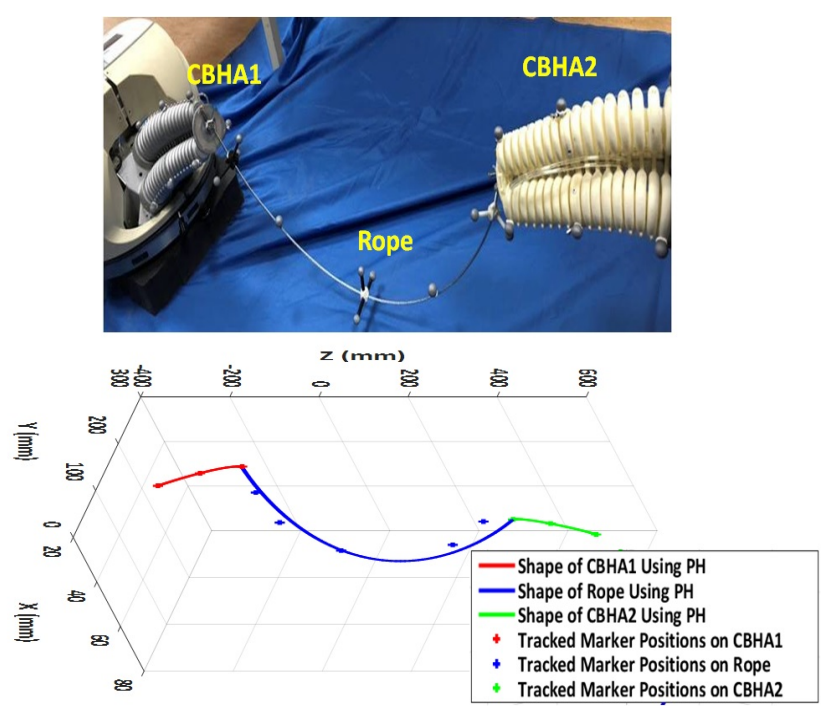

Fig. 14. Comparison of real shape with shape reconstructed using PH curve for Heterogeneous continuum closed loop kinematic chain

movements of the kinematic chain are created as shown in the attached video. Using the tracking system, data is recorded for some of the postures during the movement. We tried to show these postures along with the movement of the kinematic chain in the video. 
TABLE II

ABSOLUTE ERRORS IN LENGTHS FOR FULL TRAJECTORY

\begin{tabular}{|c|c|c|c|}
\hline & Min Err in L1 $(\mathrm{mm})$ & Max Err in L1 $(\mathrm{mm})$ & Avg Err in L1 $(\mathrm{mm})$ \\
\hline Pythagorean Hodograph Curves Approach & 0.0112 & 4.4020 & 2.8239 \\
\hline Newton Raphson Approach & 1.9555 & 13.2310 & 6.3947 \\
\hline Hybrid Approach & 8.6865 & 24.1922 & 14.5922 \\
\hline & Min Err in L2 $(\mathrm{mm})$ & Max Err in L2 (mm) & Avg Err in L2 (mm) \\
\hline Pythagorean Hodograph Curves Approach & 0.0032 & 5.2327 & 2.2020 \\
\hline Newton Raphson Approach & 0.1101 & 7.5254 & 3.9361 \\
\hline Hybrid Approach & 2.7561 & 7.4398 & 4.9772 \\
\hline & Min Err in L3 (mm) & Max Err in L3 (mm) & Avg Err in L3 (mm) \\
\hline Pythagorean Hodograph Curves Approach & 0.0012 & 3.5959 & 0.9768 \\
\hline Newton Raphson Approach & 0.0054 & 9.9292 & 6.2398 \\
\hline Hybrid Approach & 0.0074 & 8.5180 & 4.3089 \\
\hline
\end{tabular}

TABLE III

TIME COST FOR ONE SAMPLE

\begin{tabular}{|c|c|}
\hline & Time Cost $(\mathrm{sec})$ \\
\hline Pythagorean Hodograph Curves Approach & 0.00027 \\
\hline Newton Raphson Approach & 0.23351 \\
\hline Hybrid Approach & 0.01208 \\
\hline
\end{tabular}

\section{CONCLUSIONS}

In this paper, a quantitative approach to model the shape reconstruction as well as kinematic behavior of a heterogeneous continuum closed-loop kinematic chain using $\mathrm{PH}$ curves is proposed and validated experimentally. Comparison with two existed models indicates the enhancement of the performances regarding time cost as well as accuracy for the same trajectory tracking. Our future work includes the $\mathrm{PH}$ model-based control of rope using two collaborative continuum robots. Controlling the shape of the rope is an important task for the application of skipping rope.

\section{ACKNOWLEDGMENT}

This work was supported by the joint Collaborative Research Project between CNRS in France and DST in India (PRC CNRS-DST 2016-2018). The authors want to thank Dr. Reinhard Pittschellis and Dr. Dirk Pensky from Festo Didactic company in Denkendorf (Germany) for their technical assistance and support on RobotinoXT.

\section{REFERENCES}

[1] R. Behrens, M. Poggendorf, E. Schulenburg, N. Elkmann. An elephant's trunk-inspired robotic arm-trajectory determination and control. In Robotics, 2012 7th German Conference: 1-5.

[2] M. Sfakiotakis, A. Kazakidi, N. Pateromichelakis, D. P. Tsakiris. Octopus-inspired eight-arm robotic swimming by sculling movements. In Robotics and Automation (ICRA), 2013 IEEE International Conference: $5155-5161$.

[3] I. S. Godage, E. Guglielmino, D. T. Branson, G. A. Medrano-Cerda, D. G. Caldwell. Novel modal approach for kinematics of multisection continuum arms. In Intelligent Robots and Systems (IROS), 2011 IEEE/RSJ International Conference: pp. 1093-1098.

[4] I. S. Godage, D. T. Branson, E. Guglielmino, G. A. Medrano-Cerda, D. G. Caldwell. Shape function-based kinematics and dynamics for variable length continuum robotic arms. In Robotics and Automation (ICRA), 2011 IEEE International Conference: 452-457.

[5] M. Rolf, J. J. Steil. Constant curvature continuum kinematics as fast approximate model for the Bionic Handling Assistant. In Intelligent Robots and Systems (IROS), 2012 IEEE/RSJ International Conference: $3440-3446$.
[6] I. Singh, O. Lakhal, R. Merzouki. Towards Extending Forward Kinematic Models on Hyper-Redundant Manipulator to Cooperative Bionic Arms. In Journal of Physics: Conference Series. 2017; 783(1): 012056

[7] D. Trivedi, A. Lotfi, C. D. Rahn. Geometrically exact models for soft robotic manipulators. IEEE Transactions on Robotics. 2008; 24(4): $773-780$.

[8] M. Giorelli, F. Renda, G. Ferri, C. Laschi. A feed-forward neural network learning the inverse kinetics of a soft cable-driven manipulator moving in three-dimensional space. In Intelligent Robots and Systems (IROS), 2013 IEEE/RSJ International Conference: 5033-5039.

[9] A. Melingui, O. Lakhal, B. Daachi, J. B. Mbede, R. Merzouki. Adaptive neural network control of a compact bionic handling arm. IEEE/ASME Transactions on Mechatronics. 2015; 20(6): 2862-2875.

[10] O. Lakhal, A. Melingui, R. Merzouki. Hybrid approach for modeling and solving of kinematics of a compact bionic handling assistant manipulator. IEEE/ASME Transactions on Mechatronics. 2016; 21(3): 1326-1335.

[11] I. Singh, O. lakhal, Y. Amara, V. Coelen, P. M. Pathak, R. Merzouki. Performances evaluation of inverse kinematic models of a compact bionic handling assistant. IEEE International Conference on Robotics and Biomimetics (ROBIO). Dec, 2017: 5-8.

[12] H. A. Wurdemann, S. Sareh, A. Shafti, Y. Noh, A. Faragasso, D. S. Chathuranga, H. Liu, S. Hirai, K. Althoefer. Embedded electroconductive yarn for shape sensing of soft robotic manipulators. In Engineering in Medicine and Biology Society (EMBC), 2015 37th Annual International Conference of the IEEE: 8026-8029.

[13] H. Wang, R. Zhang, W. Chen, X. Liang, R. Pfeifer. Shape detection algorithm for soft manipulator based on fiber bragg gratings. IEEE/ASME Transactions on Mechatronics. 2016; 21(6): 2977-2982.

[14] H. Liu, A. Farvardin, R. Grupp, R. J. Murphy, R. H. Taylor, I. Iordachita, M. Armand. Shape tracking of a dexterous continuum manipulator utilizing two large deflection shape sensors. IEEE sensors journal. 2015; 15(10): 5494-5503.

[15] H. Mochiyama, E. Shimemura and H. Kobayashi. Shape correspondence between a spatial curve and a manipulator with hyper degrees of freedom. In Intelligent Robots and Systems (IROS), 1998 IEEE/RSJ International Conference: 161-166.

[16] G. S. Chirikjian, J. W. Burdick. A modal approach to hyper-redundant manipulator kinematics. IEEE Transactions on Robotics and Automation. 1994; 10(3): 343-354.

[17] R. T. Farouki, T. Sakkalis. Pythagorean hodographs. IBM Journal of Research and Development. 1990; 34(5): 736-752.

[18] I. Singh, Y. Amara, A. Millengui, P. M. Pathak, R. Merzouki. Modeling of continuum manipulators using pythagorean hodograph curves. Soft Robotics, 2018.

[19] R. T. Farouki. Pythagorean-hodograph curves: algebra and geometry inseparable (Vol. 1), Springer Science \& Business Media: 2008.

[20] R. Dietz, J. Hoschek, B. Jttler. An algebraic approach to curves and surfaces on the sphere and on other quadrics. Computer Aided Geometric Design. 1993; 10(3-4): 211-229.

[21] R. T. Farouki, M. al-Kandari, T. Sakkalis. Hermite interpolation by rotation-invariant spatial Pythagorean-hodograph curves. Advances in Computational Mathematics. 2002; 17(4): 369-383.

[22] https://www.festo.com/group/en/cms/10239.htm. 